\title{
Detection of Leptin Activity in Living Cells Expressing Chicken Leptin Receptor and STAT3
}

\author{
Hiromi Adachi ${ }^{1,2}$, Daisuke Murase ${ }^{1,2}$, Susumu Atomura ${ }^{1}$ and Takeshi Ohkubo ${ }^{1}$ \\ ${ }^{1}$ College of Agriculture, Ibaraki University, 3-21-1 Chuo, Ami, Ibaraki 300-0393, Japan \\ ${ }^{2}$ United Graduate School of Agriculture, Tokyo University of Agriculture and Technology, \\ 3-8-1 Harumi, Fuchu, 183-8538 Tokyo, Japan
}

\begin{abstract}
The chicken leptin receptor (chLEPR) activates Janus kinase (JAK) - signal transducers and activator of transcription (STAT) signaling pathway after leptin stimulation. We have previously developed a bioassay using leptin inducible reporter gene in cultured cells. However, we failed to detect leptin-like activity in chicken blood. In the present study, we expressed green fluorescent protein (GFP) fused chSTAT3 (GFP-chSTAT3) in cells expressing chLEPR and analyzed leptin dependent activation of the chSTAT3. Leptin phosphorylated GFP-chSTAT3 and by which fluorescent signal translocated into nuclei in COS-7 cells transiently expressing GFP-chSTAT3 with chLEPR. Furthermore, we established CHO-K1 cells stably expressing chLEPR and chSTAT3 (CHO-chLEPR/chSTAT3), and in which detected time- and dose-dependent activation of chSTAT3 by leptin. Therefore, the CHO-chLEPR/STAT3 cells would be an excellent tool to detect and monitor leptin-like activity in avian tissues.
\end{abstract}

Key words: bioassay, chicken, leptin, signal transduction, STAT3

J. Poult. Sci., 49: 46-50, 2012

\section{Introduction}

The chicken (ch) leptin receptor (chLEPR) was cloned by our laboratory and others (Horev et al., 2000; Ohkubo et al., 2000 ) as the first LEPR cloned from non-mammalian vertebrates. In addition, chLEPR gene has been mapped on the chromosome 8 of chicken genome (Dunn et al., 2000). Beside existence of chLEPR gene, identification of chicken leptin gene is still in conflict (Friedman-Einat et al., 1999; Amills et al., 2003, Richards and Proszkowiec-Weglarz, 2007). Nevertheless the evidence for the expression of functional LEPR in avian target tissues comes from the observation that exogenous leptin treatment modulates physiological process including food intake, immune response and ovarian function in birds (Ohkubo and Adachi, 2008, Review). In addition, infusion of radiolabeled leptin to chicken led to detection of radioactivity in multiple tissues (McMurtry et al., 2004). Western blot analysis has identified the presence of LEPR protein in the chicken brain and chicken hepatoma cell, LMH (Ohkubo et al., 2007).

Following leptin binding with LEPR, activated Janus kinase 2 (JAK2) rapidly phosphorylates signal transducers

Received: August 10, 2011, Accepted: October 26, 2011

Released Online Advance Publication: November 25, 2011

Correspondence: T. Ohkubo, College of Agriculture, Ibaraki University, 3-21-1 Chuo, Ami, Ibaraki 300-0393, Japan.

(E-mail: ohkubo@mx.ibaraki.ac.jp) and activator of transcription 3 (STAT3). Phosphorylated STAT3 then translocates to the nucleus to modulate transcription of the target genes in mammals (Banks et al., 2000). Recently it has been shown by us and others that chLEPR expressed in mammalian cells activates JAK - STAT signaling pathway (Adachi et al., 2008; Hen et al., 2008), indicating thereby that endogenous LEPR in bird is capable of processing leptin-like signals in a way similar to higher vertebrates. However, it has not been possible to detect leptin-like activity in the avian blood by the in vitro bioassay (Adachi et al., 2008; Hen et al., 2008; Yosefi et al., 2010). This may be due to plasma leptin levels being below the detection threshold of the quantitative bioassay using luciferase gene. Therefore, the possibility that there is no natural ligand of the LEPR in birds cannot be completely ruled out.

An alternative way to detect endogenous leptin-like activity in birds is to monitor intracellular localization of STAT3. It is known that fluorescent protein fused STAT3 migrates into nucleus after cytokine stimulation (Kretzschmar et al., 2004; Pranada et al., 2004; Watanabe et al., 2004). In addition, leptin activates chLEPR which induces STAT dependent activation of luciferase gene (Adachi et al., 2008; Hen et al., 2008). Therefore, detection of translocation of GFP fused STAT3 into nuclei would be an alternate bioassay in detecting endogenous leptin-like activity in birds. To test this possibility, we expressed chicken STAT3 as GFP tagged protein and then detecting its translocation into nuclei. 


\section{Materials and Methods}

\section{Antibodies}

Anti-STAT3 monoclonal antibody and anti-mouse STAT3 (pY705) monoclonal antibody were obtained from BD Biosciences (Tokyo, Japan). These antibodies were suitable for the detection chicken STAT3 (Adachi et al., 2008). AntiGFP monoclonal antibody was purchased from Nacalai Tesque (Kyoto, Japan). Anti-chLEPR monoclonal antibody was described previously (Ohkubo et al., 2007).

\section{Plasmids and Vector Construction}

An expression vector for chicken leptin receptor (pCIchLEPR) has been described previously (Ohkubo et al., 2007). Each pGEM-T Easy, pcDNA3. 1Hygro $(+)$ and pEGFP-N1 was obtained from Promega (Tokyo, Japan), Invitrogen (Tokyo, Japan) and Clontech (Palo Alto, CA, USA), respectively.

The entire coding sequence of chicken STAT3 (chSTAT3) was amplified by PCR using a pair of primers, $5^{\prime}$-GCTCGAGGATGGCGCAGTGGAACCA-3' (sense: nucleotide 1835 of NM_001030931 with Xho I site) and 5' -TGGATCCATTGGTGÄGGAAGCACACT-3' (antisense: nucleotide 23122331 of NM_001030931 with Eco RI site) with White Leghorn hypothalamic cDNA library (Ohkubo et al., 2000). PCR was carried out for 25 cycles of $95^{\circ} \mathrm{C}$ for $30 \mathrm{~s}, 60^{\circ} \mathrm{C}$ for $30 \mathrm{~s}$, and $72^{\circ} \mathrm{C}$ for $2 \mathrm{~min} 30 \mathrm{~s}$. Thereafter, the amplified fragment was cloned into pGEM-T Easy (Promega) and sequenced. This was followed by introduction of chSTAT3 cDNA into pEGFP-N1 (pEGFP-chSTAT3). Subsequently, chLEPR cDNA removed from pCI-chLEPR was cloned into pcDNA3.1 Hygro $(+)$ (pcDNA-chLEPR).

\section{Cell Culture}

CHO-K1 cell and CHO-K1 cell stably expressing chLEPR (CHO-chLEPR) (Adachi et al., 2008) were maintained in nutrient mixture Ham's F-12 medium (Sigma-Aldrich Japan, Tokyo, Japan) containing 10\% fetal bovine serum (FBS), $100 \mathrm{units} / \mathrm{m} l$ penicillin and $100 \mu \mathrm{g} / \mathrm{m} l$ streptomycin at $37^{\circ} \mathrm{C}$ in an atmosphere of $95 \%$ air and $5 \% \mathrm{CO}_{2}$. COS-7 cell was maintained in Dulbecco's modified Eagle's medium supplemented with $10 \% \mathrm{FBS}, 100 \mathrm{units} / \mathrm{m} l$ penicillin and $100 \mu \mathrm{g} / \mathrm{ml}$ streptomycin under $5 \% \mathrm{CO}_{2}$ and $95 \%$ air at $37^{\circ} \mathrm{C}$.

\section{Expression of GFP-fused chSTAT3 in COS-7 Cells}

COS-7 cell grown in $35 \mathrm{~mm}^{2}$ dish $\left(3.0 \times 10^{5}\right.$ cells/well $)$ was transfected with $2 \mu \mathrm{g}$ of pEGFP-chSTAT3 or pEGFP-N1 as control using TransFectin Lipid Reagent (Bio-Rad Laboratories, Tokyo, Japan) according to the manufacturer's instructions. Twenty-four hours after the transfection, cells were harvested and lysed with $100 \mu l$ of Mild Lysis Solution (CytoSignal, Irvine, CA, USA). Protein concentration of the cell lysates was measured by using Pierce BCA Protein Assay Kit (TaKaRa Bio, Shiga, Japan) and these were stored at $-20^{\circ} \mathrm{C}$ until used for Western blot analysis for STAT3 and GFP.

Leptin Dependent Activation of GFP-fused chSTAT3 in COS-7 Cells

COS-7 cells seeded in 24 well plate $\left(0.8 \times 10^{5}\right.$ cells/well $)$ were transfected with $0.25 \mu \mathrm{g}$ each of pCI-chLEPR and
pEGFP-chSTAT3 by TransFectin Lipid Reagent (Bio-Rad Laboratories) under manufacuter's protocol. These were then incubated for $4 \mathrm{~h}$ and again for another $16 \mathrm{~h}$ in serum free medium. Subsequently the cells were treated with 125 $\mathrm{ng} / \mathrm{m} l$ recombinant murine leptin (rmleptin ; Sigma Aldrich Japan, Tokyo, Japan) for 10, 30, 60 and $120 \mathrm{~min}$. At the end of each treatment, cells were harvested and stored at $-20^{\circ} \mathrm{C}$ until use for Western blot analysis for STAT3.

\section{Western Blot Analysis}

Total cell lysates were separated by SDS-PAGE under reducing conditions and then transferred to Hybond-P membrane (GE Healthcare Japan, Tokyo, Japan) using TransBlot SD semi-dry transfer cells (Bio-Rad Laboratories) at 15 $\mathrm{V}$ for $30 \mathrm{~min}$ in $25 \mathrm{mM}$ Tris, $192 \mathrm{mM}$ glycine, and $20 \%$ methanol. After transfer, the blot was treated with PBS containing $0.1 \%$ Tween 20 (PBST) including 5\% non-fat dry milk for $1 \mathrm{~h}$ at room temperature. The membrane was incubated with appropriate primary antibody for $1 \mathrm{~h}$ at room temperature followed by washing with PBST for $10 \mathrm{~min}$, twice. Then the blot was with secondary antibody conjugated with horseradish peroxidase for $1 \mathrm{~h}$ and washed with PBST for 10 min, twice. After washing, the membrane was then immersed in SuperSignal West Dura Extended Duration Substrate (TaKaRa Bio) and the signals detected by LAS3000 mini (Fuji Film Co. Ltd., Tokyo, Japan).

Determination of Leptin Induced Nucleus Accumulation of chSTAT3 in COS-7 Cells Transiently Expressing chLEPR

Seeding of $0.8 \times 10^{5}$ of COS-7 cells were made in 24 well plate dish with a glass coverslip and were transfected with 0 . $25 \mu \mathrm{g}$ of pcDNA-chLEPR and pEGFP-chSTAT3 using TransFectin Lipid Reagent (Bio-Rad Laboratories). Four hours after transfection, the medium was replaced by serumfree medium and was left for $16 \mathrm{~h}$. Then the cells were stimulated with $125 \mathrm{ng} / \mathrm{m} l$ of rmleptin (Sigma-Aldrich Japan) for $0,10,30,60$ and $120 \mathrm{~min}$. After the stimulation, the cells were fixed in $4 \%$ formaldehyde and stained with 2-(4amidinophenyl)- $1 H$-indole-6-carboxamidine (DAPI). Then, the coverslip was mounted on a glass slide, and the fluorescent signal was detected by a fluorescence microscope (Nikon Labophot, Nikon, Tokyo, Japan).

Determination of Leptin Induced Intra-nucleus Translocation of chSTAT3 in CHO-K1 Cells Stably Expressing GFP-tagged chSTAT3 with chLEPR

Stable cell line expressing chLEPR and GFP-fused chSTAT3 was established by transfecting CHO-K1 cells with each 0.25 $\mu \mathrm{g}$ of pcDNA-chLEPR and pEGFP-chSTAT3 using TransFectin Lipid Reagent (Bio-Rad Laboratories). Twenty-four hours after transfection, the cells were exposed to a medium containing $500 \mu \mathrm{g} / \mathrm{ml}$ G418 (Nacalai Tesque, Kyoto, Japan) and $200 \mu \mathrm{g} / \mathrm{m} l$ of HygroGold (InvivoGen, San Diego, CA, USA). Cells expressing both chLEPR and GFP-tagged chSTAT3 were selected by Western blot using anti-chLEPR monoclonal antibody and anti-STAT3 monoclonal antibody, and established cells stably expressing chLEPR and chSTAT3 (CHO-chLEPR/STAT3). At the same time, CHO-K1 cells stably expressing GFP-fused chSTAT3 were constructed as control of CHO-chLEPR/STAT3. 
The CHO-chLEPR/STAT3 were treated with rmleptin, and nuclear accumulation of chSTAT3 was monitored for $45 \mathrm{~min}$ by BZ-1000 All-in-One Fluorescence Microscope (KEYENCE Japan, Tokyo, Japan).

\section{Results}

\section{Expression of GFP Tagged chSTAT3 in COS-7 Cell}

GFP-tagged chSTAT3 was detected as approximately 110 $\mathrm{kDa}$ protein by Western blot analysis with anti-STAT3 and anti-GFP antibodies (Fig. 1). The molecular weight of the protein was similar to the estimated molecular mass of chSTAT3 with GFP.

\section{Leptin Dependent Activation of GFP-fused chSTAT3 in} COS-7 Cells

In Western blot analysis, it was found that leptin phosphorylated the GFP-chSTAT3 in COS-7 cells expressing chLEPR. The phosphorylated chSTAT3 was detected in 10 min and this is observed by 2 hours after stimulation (Fig. 2).

In fluorescence microscopic analysis, green fluorescence dispersed in the cytoplasm in COS-7 cells co-transfected with GFP-chSTAT3 and pCI-chLEPR as well as in control cells before leptin stimulation (Fig. 3). Leptin induced translocation of GFP-chSTAT3 into nuclei was detected by 10 min and intranuclear chSTAT3 was found by $2 \mathrm{~h}$ after leptin treatment. However, green fluorescence in the COS-7 cells exposed $2 \mathrm{~h}$ with rmleptin was found in the cytoplasm as well

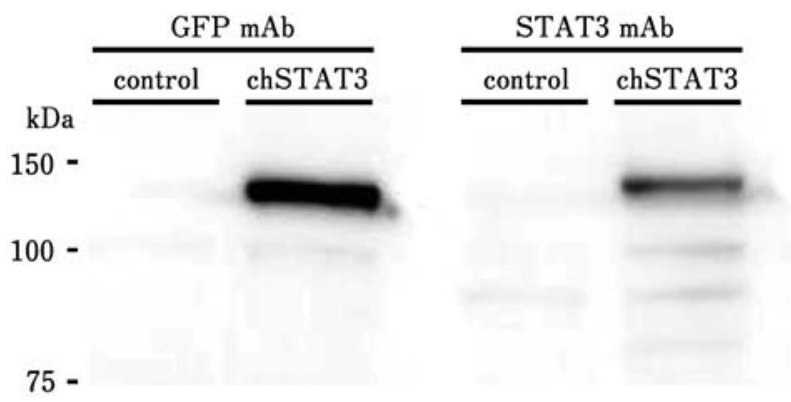

Fig. 1. Western blot analysis of GFP fused chSTAT3 expressed in COS-7 cells. Whole cell lysates prepared from COS-7 transfected with pEGFP-N1 (control) or pEGFPchSTAT3 (chSTAT3) were subjected to Western blot using anti-GFP monoclonal antibody (GFP mAb) or anti-STAT3 monoclonal antibody (STAT3 mAb).

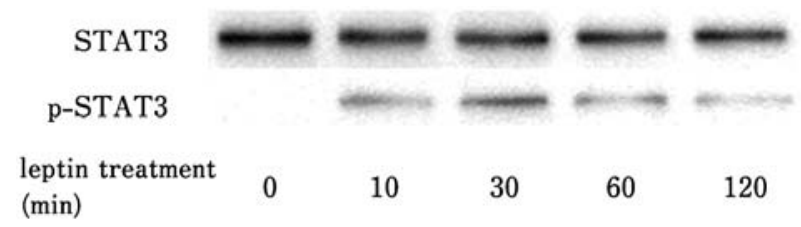

Fig. 2. Phosphorylation of GFP-fused chSTAT3 by leptin stimulation. COS-7 transfected with pEGFP-chSTAT3 was treated with $125 \mathrm{ng} / \mathrm{m} l \mathrm{rmleptin}$. Whole cell lysates prepared at $0,10,30,60$ and 120 min after leptin treatment was analyzed by Western blot using anti-STAT3 monoclonal antibody and anti-mouseSTAT3 (pY705) monoclonal antibody. as in the nuclei (Fig. 3), which may indicate increased inactivated or dephosphorylated form of chSTAT3 in the cells. Activation of chSTAT3 by leptin in CHO-K1 cells stably expressing GFP-fused chSTAT3.

Green fluorescence signals accumulated into nucleus after rmleptin stimulation in CHO-chLEPR/STAT3 cells, whereas no such dynamic change was found in CHO-chSTAT3 cells (Fig. 4). Frequency of nucleus condensation of the GFP signals was more with stimulation by $10 \mathrm{ng} / \mathrm{ml}$ of rmleptin than by $5 \mathrm{ng} / \mathrm{ml}$ (Fig. 4).

\section{Discussion}

Since identification of chicken leptin gene is still in conflict (Friedman-Einat et al., 1999; Amills et al., 2003; Richards and Proszkowiec-Weglarz, 2007), we had earlier established a bioassay to detect leptin activity using chicken LEPR (Adachi et al., 2008). However, we could not detect leptin-like activity in chicken serum. Other researchers had also failed to detect serum leptin activity in chicken and other birds using similar bioassay (Hen et al., 2008; Yosefi et al., 2010).

STAT proteins are mediators of cytokine-regulated genes and exist in cytoplasm as monomers. STATs are phosphorylated by JAK after cytokine stimulation, and activated STATs dimerize and translocate into nucleus. The use of GFP as a tag for shuttling protein is valuable to study the trafficking of signaling molecule in individual living cells (Cubitt et al., 1995; Htun et al., 1996; Misteli et al., 1997). In the present study, we developed a new bioassay using GFP tagged chSTAT3 to detect leptin activity in living cells. GFP-fused chSTAT3 was transiently expressed in COS-7 cells (Fig. 1) and was phosphorylated after leptin treatment (Fig. 2). In addition, intracellular localization of GFP-chSTAT3 changed after leptin treatment. After leptin stimulation, fluorescence signals were observed around cell membrane as well as in nuclei in $10 \mathrm{~min}$ after leptin stimulation and intranuclear GFP-chSTAT3 was observed by $2 \mathrm{~h}$ after leptin treatment (Fig. 3). However, most of the signals condensed in the nuclei spread to the cytoplasm $2 \mathrm{~h}$ after leptin treatment (Fig. 3). It is suggested that activated STAT6, which belongs to the same STAT family with STAT3, is deactivated by dephosphorylation and which initiates recruitment of the molecule (Andrews et al., 2002). Data obtained from the fluorescence microscopic analysis may therefore reflect physiological process of STAT3 after leptin stimulation. In addition, we observed that GFP-chSTAT3 activated STAT3responsive reporter gene on stimulation by leptin (data not shown). Therefore, GFP-chSTAT3 acts as transcription factor in activating target genes. We then therefore established cells stably expressing chLEPR and GFP-chSTAT3 (CHOchLEPR/STAT3) to develop a new bioassay monitoring leptin activity using fluorescence microscope in living cells. Leptin specifically induced nuclear transition of GFPchSTAT3 in CHO-chLEPR/STAT3 in a dose dependent manner (Fig 4). Reporter gene assays developed by us and others require a long time to determine specific leptin activity (Adachi et al., 2008; Hen et al., 2008), and it is noteworthy 

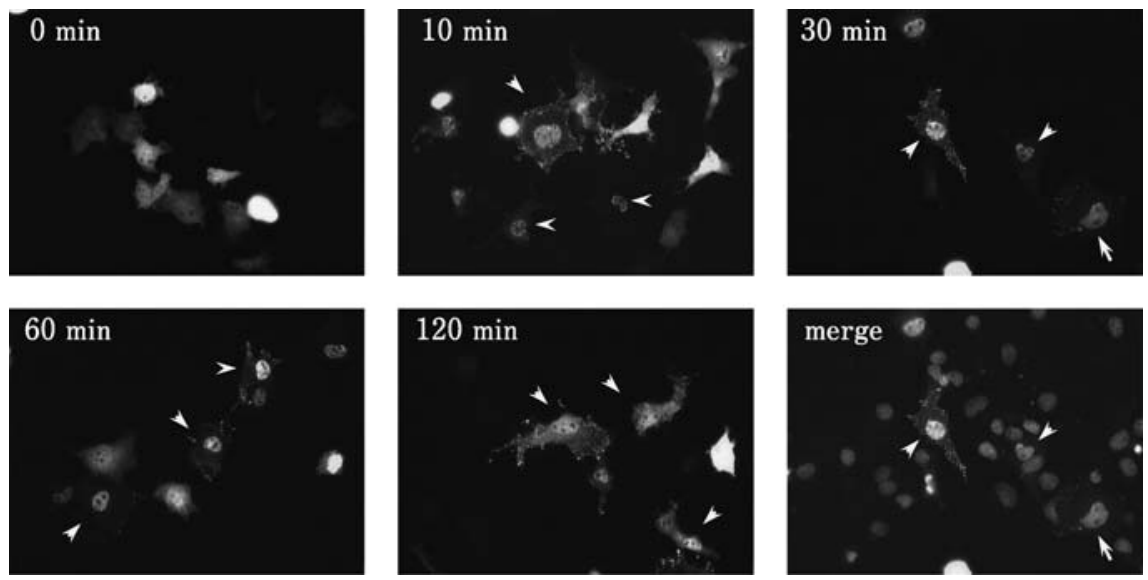

Fig. 3. Cellular localization of GFP-fused chSTAT3 after leptin stimulation in COS-7 cells. COS-7 transfected with pEGFP-chSTAT3 was stimulated with 125 $\mathrm{ng} / \mathrm{m} l$ of rmleptin for $0,10,30,60$ and $120 \mathrm{~min}$. Arrowhead indicates chSTAT3 migrated into nucleus by leptin stimulation. Nucleus condensation of the STAT3 was confirmed by DAPI stain. The representative photo confirming condensation of STAT3 in the nucleus after 30 min rmleptin stimulation is shown as merge.
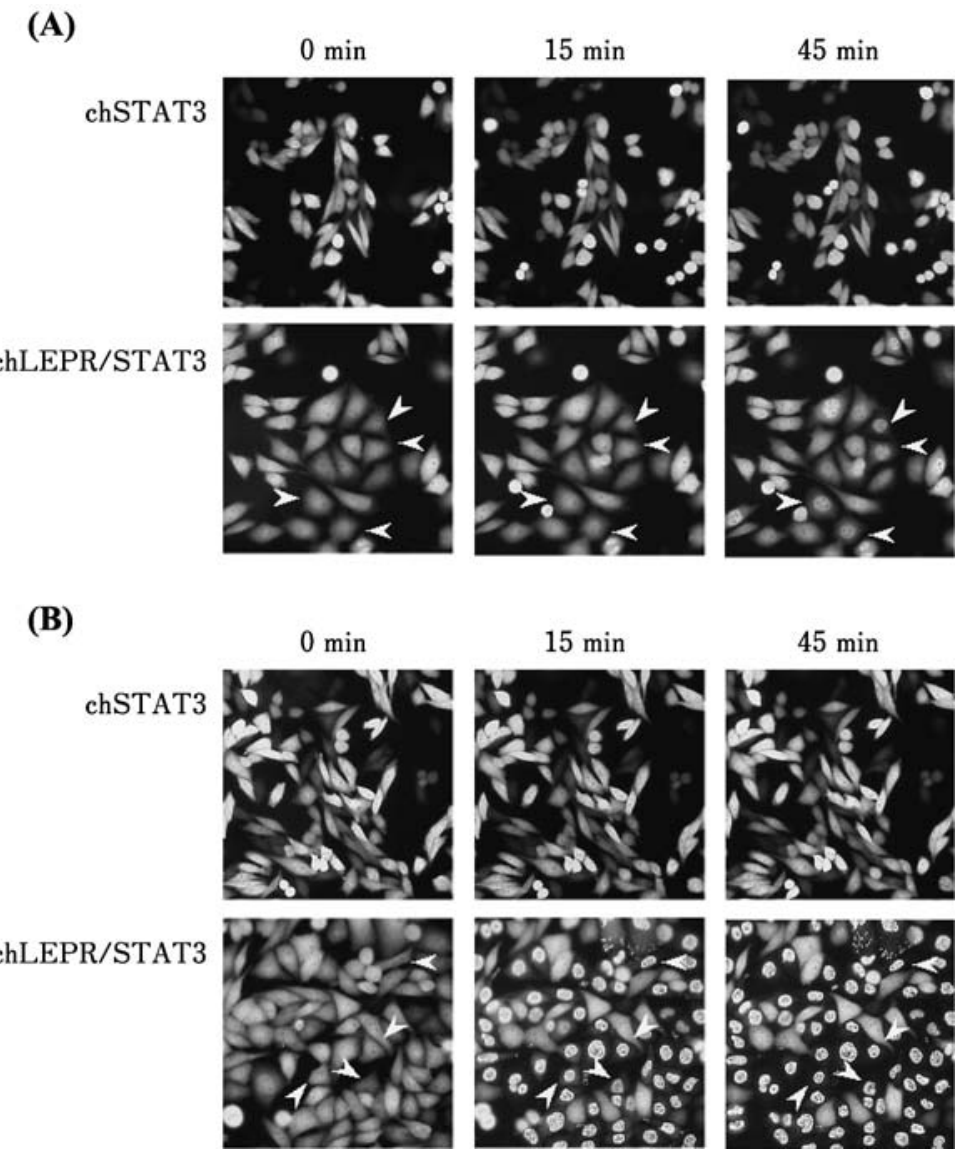

Fig. 4. Time dependent change of intracellular GFP-fused chSTAT3 by rmleptin in CHO-chLEPR/STAT3 cells. CHO-chLEPR/chSTAT3 (chLEPR/ STAT3) and CHO-chSTAT3 (chSTAT3) as control were stimulated with $5 \mathrm{ng} / \mathrm{m} l$ (A) or $10 \mathrm{ng} / \mathrm{ml}$ (B) rmleptin and in which intracellular behavior of GFP-fused chSTAT3 was monitored by fluorescence microscope. Arrowhead indicates cells chSTAT3 was activated by leptin treatment. 
that in the present study, leptin dependent activation of STAT3 was found in less than one hour.

Physiological leptin concentrations in blood of mammals range from 3 to $80 \mathrm{ng} / \mathrm{ml}$ (Tomimatsu et al., 1997; FriedmanEinat et al., 2003; Nkrumah et al., 2007). We confirmed that rat plasma specifically activated chLEPR in CHOchLEPR/STAT3 cells (data not shown). This result indicates that CHO-chLEPR/STAT3 cells seem to be sensitive to the physiological dose of leptin in mammals. As xenopus leptin activates chLEPR (Hen et al., 2008), it is likely that CHOchLEPR/STAT3 specifically binds with leptin-like molecule in lower vertebrates. Therefore, CHO-chLEPR/STAT3 would be useful as a qualitative bioassay to determine leptin-like activity in blood and tissues in vertebrates including birds. Further experiment is required to confirm whether leptinlike activity is detected from avian derived sample using this bioassay system to rule out existence of leptin-like molecules in bird.

In conclusion, we established a cell stably expressing chicken LEPR and GFP-tagged chSTAT3 and this makes it possible to determine leptin-like activity in living cells. Therefore, the CHO-chLEPR/STAT3 cells are excellent tools to detect and monitor leptin-like activity in avian tissues.

\section{Acknowledgment}

This study was supported in part by a Grant-in-Aid for Scientific Research (C) from the Promotion of Sciences to T.O. (No. 18580290) and Research Fellowship of the Japan Society for the Promotion of Science for Young Scientists to H.A.

\section{References}

Adachi H, Takemoto Y, Bungo T and Ohkubo T. Chicken leptin receptor is functional in activating JAK-STAT pathway in vitro. Journal of Endocrinology, 197: 335-342. 2008.

Amills M, Jimenez N, Villalba D, Tor M, Molina E, Cubilo D, Marcos C, Francesch A, Sanchez A and Estany J. Identification of three single nucleotide polymorphisms in the chicken insulin-like growth factor 1 and 2 genes and their associations with growth and feeding traits. Poultry Science, 82: 14851493. 2003.

Andrews RP, Ericksen MB, Cunningham CM, Daines MO and Hershey GK. Analysis of the life cycle of stat6. Continuous cycling of STAT6 is required for IL-4 signaling. Journal of Biological Chemistry, 277: 36563-36569. 2002.

Banks AS, Davis SM, Bates SH and Myers MG Jr. Activation of downstream signals by the long form of the leptin receptor. Journal of Biological Chemistry, 275: 14563-14572. 2000.

Cubitt AB, Heim R, Adams SR, Boyd AE, Gross LA and Tsien RY. Understanding, improving and using green fluorescent proteins. Trends in Biochemical Sciences, 20: 448-455. 1995.

Dunn IC, Boswell T, Friedman-Einat M, Eshdat Y, Burt DW and Paton IR. Mapping of the leptin receptor gene (LEPR) to chicken chromosome 8. Animal Genetics, 31: 290. 2000.

Friedman-Einat M, Boswell T, Horev G, Girishvarma G, Dunn IC, Talbot RT and Sharp PJ. The chicken leptin gene: has it been cloned? General and Comparative Endocrinology, 115: 354363. 1999.

Friedman-Einat M, Camoin L, Faltin Z, Rosenblum CI, Kaliouta V, Eshdat Y and Strosberg AD. Serum leptin activity in obese and lean patients. Regulatory Peptides, 111: 77-82. 2003.

Hen G, Yosefi S, Ronin A, Einat P, Rosenblum CI, Denver RJ and Friedman-Einat M. Monitoring leptin activity using the chicken leptin receptor. Journal of Endocrinology, 197: 325-333. 2008.

Horev G, Einat P, Aharoni T, Eshdat Y and Friedman-Einat M. Molecular cloning and properties of the chicken leptinreceptor (CLEPR) gene. Molecular and Cellular Endocrinology, 162: 95-106. 2000.

Htun H, Barsony J, Renyi I, Gould DL and Hager GL. Visualization of glucocorticoid receptor translocation and intranuclear organization in living cells with a green fluorescent protein chimera. Proceedings of the National Academy of Sciences of the United States of America, 93: 4845-4850. 1996.

Kretzschmar AK, Dinger MC, Henze C, Brocke-Heidrich K and Horn F. Analysis of Stat3 (signal transducer and activator of transcription 3) dimerization by fluorescence resonance energy transfer in living cells. Biochemical Journal, 377: 289-297. 2004.

McMurtry JP, Ashwell CM, Brocht DM and Caperna TJ. Plasma clearance and tissue distribution of radiolabeled leptin in the chicken. Comparative Biochemistry and Physiology Part A, Molecular \& Integrative Physiology, 138: 27-32. 2004.

Misteli T, Caceres JF and Spector DL. The dynamics of a premRNA splicing factor in living cells. Nature, 387: 523-527. 1997.

Nkrumah JD, Keisler DH, Crews DH Jr, Basarab JA, Wang Z, Li C, Price MA, Okine EK and Moore SS. Genetic and phenotypic relationships of serum leptin concentration with performance, efficiency of gain, and carcass merit of feedlot cattle. Journal of Animal Science, 85: 2147-2155. 2007.

Ohkubo $\mathrm{T}$ and Adachi H. Leptin signaling and action in birds. Journal of Poultry Science, 45: 233-240. 2008.

Ohkubo T, Nishio M, Tsurudome M, Ito M and Ito Y. Existence of leptin receptor protein in chicken tissues: isolation of a monoclonal antibody against chicken leptin receptor. General and Comparative Endocrinology, 151: 269-273. 2007.

Ohkubo T, Tanaka M and Nakashima K. Structure and tissue distribution of chicken leptin receptor (cOb-R) mRNA. Biochimica et Biophysica Acta, Gene Structure and Expression, 1491: 303-308. 2000.

Pranada AL, Metz S, Herrmann A, Heinrich PC and MüllerNewman G. Real time analysis of STAT3 nucleocytoplasmic shuttling. Journal of Biological Chemistry, 279: 15114-15123. 2004.

Richards MP and Proszkowiec-Weglarz M. Mechanisms regulating feed intake, energy expenditure, and body weight in poultry. Poultry Science, 86: 1478-1490. 2007.

Tomimatsu T, Yamaguchi M, Murakami T, Ogura K, Sakata M, Mitsuda N, Kanzaki T, Kurachi H, Irahara M, Miyake A, Shima K, Aono T and Murata Y. Increase of mouse leptin production by adipose tissue after midpregnancy: gestational profile of serum leptin concentration. Biochemical and Biophysical Research Communications, 240: 213-215. 1997.

Watanabe K, Saito K, Kinjo M, Matsuda T, Tamura M, Kon S, Miyazaki T and Ueda T. Molecular dynamics of STAT3 on IL6 signaling pathway in living cells. Biochemical and Biophysical Research Communications, 324: 1264-1273. 2004.

Yosefi S, Hen G, Rosenblum CI, Cerasale DJ, Beaulieu M, Criscuolo F and Friedman-Einat M. Lack of leptin activity in blood samples of Adelie penguin and bar-tailed godwit. Journal of Endocrinology, 207: 113-122. 2010. 